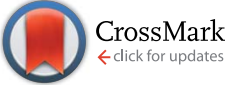

Cite this: RSC Adv., 2017, 7, 1271

\title{
Antioxidative, membrane protective and antiapoptotic effects of melatonin, in silico study of physico-chemical profile and efficiency of nanoliposome delivery compared to betaine
}

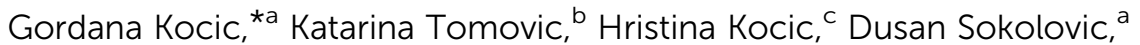 \\ Branka Djordjevic, ${ }^{a}$ Svetlana Stojanovic, ${ }^{a}$ Ivana Arsic ${ }^{b}$ and Andrija Smelcerovic ${ }^{\text {bd }}$
}

The mechanisms through which melatonin exerts its inhibitory effect on cell death remains insufficiently clarified. The subject of the present study is the evaluation of the hepatoprotective effects of melatonin on the inhibition of apoptotic and oxidative processes and activation of survival pathways, in comparison with betaine, in a primary culture of hepatocytes that have undergone Fas-ligand apoptosis. Melatonin exerted a protective effect on membrane bilayer stability through the inhibition of phosphatidylserine externalization, Bax expression and xanthine oxidase catalyzed free-radical liberation. It also reduced liberation of cellular and membrane-bound enzymes and endonuclease catalyzed DNA fragmentation. Betaine hydrochloride did not exert these effects, when administered alone or co-incubated with antiFas antibodies. The in silico Molinspiration tool, which was employed to calculate melatonin and betaine physico-chemical properties and membrane interaction indicated that melatonin may easily cross and interact with biological membranes and maintain membrane phospholipid structural topography. It was documented to occur via non-receptor mechanisms in more than $75 \%$; this may clarify melatonin as suitable for nanoliposome-based delivery, where it was also able to successfully counteract induced oxidative stress. This could not be considered for betaine hydrochloride. The use of lipophilic compounds like melatonin, encapsulated in nanoliposomes, could therefore be a preferable tool in the successful membrane-preservation therapy of liver apoptosis, rather than the use of hydrophilic compounds, like betaine hydrochloride.

Received 4th October 2016 Accepted 25th November 2016 DOI: 10.1039/c6ra24741e

www.rsc.org/advances potentially hepatoprotective substances. ${ }^{5}$ The binding of specific ligands to Fas receptors causes a cascade of signaling effects following the activation of executor caspase and endonuclease enzymes. ${ }^{1,2}$ Cells undergoing Fas-mediated apoptosis such as hepatocytes and T lymphocytes show PS externalization and subsequent clearance by phagocytes before lysis, or inflammatory reaction. ${ }^{6}$ In an intrinsic apoptotic pathway the Bcl-2 family of proteins are potent regulators, where Bcl-2 and Bcl-xL (group I) possess antiapoptotic activity, whereas members of group II (Bax and Bak) and group III (Bid) promote cell death. ${ }^{7}$ Alteration of membrane structural stability is the mechanism responsible for the translocation of Bax to the external mitochondrial membrane leaflet, when apoptosis is induced. $^{8}$

Melatonin ( $N$-acetyl-5-methoxytriptamine) is a compound derived from L-tryptophan. ${ }^{7}$ It is a lipophilic molecule, which to some extent may freely cross cell membranes and enter cells. Moreover, it can also bind to plasma membrane melatonin receptors $\left(\mathrm{MT}_{1}\right.$ and $\left.\mathrm{MT}_{2}\right)$, members of the seven transmembrane $\mathrm{G}$ protein-coupled receptors, initiating downstream cell signaling. ${ }^{9,10}$ It has been documented that melatonin exerts
${ }^{a}$ Institute of Biochemistry, Faculty of Medicine, University of Nis, Bulevar Dr Zorana Djindjica 81, 18000 Nis, Serbia. E-mail: kocicrg@yahoo.co.uk; Fax: +381 18423 8770; Tel: +381184570029

${ }^{b}$ Department of Pharmacy, Faculty of Medicine, University of Nis, Bulevar Dr Zorana Djindjica 81, 18000 Nis, Serbia

${ }^{c}$ Faculty of Medicine, University of Maribor, Magdalenski trg 5, Maribor 2000, Slovenia ${ }^{d}$ Department of Chemistry, Faculty of Medicine, University of Nis, Bulevar Dr Zorana Djindjica 81, 18000 Nis, Serbia 
a broad range of physiological ${ }^{9}$ and protective antioxidant effects, preventing the peroxidation of membrane lipids and protecting the proteins and DNA from oxidative damage induced by hydroxyl radical $\left({ }^{\circ} \mathrm{OH}\right)$, peroxynitrite anion $\left(\mathrm{ONOO}^{-}\right)$, hydrogen peroxide $\left(\mathrm{H}_{2} \mathrm{O}_{2}\right)$, superoxide anion radical $\left(\mathrm{O}_{2}{ }^{-}\right)$or singlet oxygen $\left({ }^{1} \mathrm{O}_{2}\right)$, produced in different in vitro and in vivo conditions. ${ }^{11,12}$ Our previous results pointed out melatonin as protector against microwave irradiation. ${ }^{\mathbf{1 3 - 1 5}}$ Like an electron donor, melatonin has been shown to increase the efficiency of the electron transport chain and, as a consequence, to reduce electron leakage and the generation of free-radicals. ${ }^{16}$ Beside direct free-radical scavenging properties, it also has been shown to have indirect antioxidative effects, through the upregulation of several antioxidative enzymes. When compared to vitamin $\mathrm{E}$, melatonin was found to be more effective in limiting lipid membrane destruction, ${ }^{\mathbf{1 2 , 1 7}}$ serving as naturally mitochondria-targeted protective molecule. ${ }^{18}$ Metabolites of melatonin such as major hepatic metabolite 6-hydroxymelatonin as well as $N^{1}$-acetyl- $N^{2}$-formyl-5-methoxykynuramine and $N^{1}$ acetyl-5-methoxykynuramine, have been shown to detoxify radicals themselves. This effect, independent of the species and the type of injury and extent of organ damage, is especially true for the liver and a variety of its pathophysiological conditions. ${ }^{19}$ Furthermore, there are data documenting the influence of melatonin on Bcl-2/Bax levels in rat peripheral blood lymphocytes $^{20}$ and pancreatic cancer cell line SW-1990. ${ }^{21}$ It was recently demonstrated that melatonin may be involved in epigenetic DNA reprogramming, decreasing the levels of unmethylated DNA promoter regions. ${ }^{22}$ It has been further proven that in liver disorders with a dysfunction of the apoptotic program, like ischemia/reperfusion injury, ${ }^{23}$ viral hepatitis, ${ }^{24}$ acetaminopheninduced acute liver failure ${ }^{25}$ or cadmium-induced hepatotoxicity, ${ }^{9}$ melatonin may enhance regenerative response.

Betaine is $\mathrm{N}$-trimethylated amino acid glycine, a quaternary ammonium compound, which exists in zwitterionic form at neutral $\mathrm{pH}$. As a positively-charged amine, it requires a proteinmediated mechanism to effectively pass the membrane-lipid barrier via lipid-associated betaine-binding protein (BetP). ${ }^{26}$ Because of its three chemically-reactive methyl groups, it has a role as a donor of methyl group in many cell pathways, such as synthesis of phospholipids, proteins and in stabilization of DNA structure through the methylation. ${ }^{27}$ It may enhance the secretion of VLDL via methylation of membrane phospholipids increasing phosphatidylcholine content. ${ }^{28}$ Betaine may be a promising therapeutic agent in relieving defects associated with alcoholic liver disease. ${ }^{29}$

The subject of the present study was the hepatoprotective effects of melatonin mediation by the inhibition of apoptotic and oxidative processes and activation of survival pathways, in comparison with betaine in the primary culture of mouse hepatocytes which had undergone Fas-ligand apoptosis. The effects of melatonin and betaine on the integrity of the membrane, phospholipid asymmetry and PS (phosphatidylserine) externalization in relation to certain biomarkers of oxidative stress and apoptosis were evaluated, as well as cellular and membrane enzymes liberation, mitochondrial Bcl-2/Bax distribution and DNA degradation. Since a nanoliposome-based drug delivery system could improve the pharmacokinetic properties of certain drugs, the efficacy of melatonin and betaine hydrochloride to counteract oxidative stress in encapsulated nanoliposomes was employed. Additionally, an in silico study by using Molinspiration ${ }^{30}$ and ACD/I-Lab software ${ }^{31}$ was performed in order to gain a better insight into the physico-chemical properties of melatonin and betaine as well as to explain obtained biological effects.

\section{Materials and methods}

\section{Chemicals used}

All chemicals were of a pro analysis degree of purity. Anti-Fas antibodies (Jo CD95) were obtained from BD Biosciences. The cell culture medium (RPMI 1640), fetal calf serum (FCS), collagenase and betaine hydrochloride were purchased from Sigma Aldrich (USA), melatonin was purchased from ICN (USA).

\section{Ethical statement}

All experimental procedures were performed in compliance with the Ethical Committee guidelines and rules for the protection of experimental animals according to the Animal Welfare Rules Republic of Serbia. The approval for the animal experiment was obtained from the Animal Welfare Committee No. 01-5518-6 (University Nis Medical Faculty) after submitting the required form for approval.

\section{Isolation of primary mouse hepatocyte culture}

The experimental study was conducted on inbred, male, threemonth-old, BALB mice (22-25 g weight) which were killed under ketalar anesthesia, and whose livers were removed according to the method explained in our previous article. ${ }^{5}$ The hepatocytes were isolated from the liver tissue by use of the collagenase technique (1 mg collagenase per ml RPMI 1640 medium). Isolation of hepatocytes was monitored by microscope and collagenase activity was inhibited by 10\% FCS/RPMI 1640 medium. The digested tissue was sieved and low-speed centrifuged (600 g at $4{ }^{\circ} \mathrm{C}$ for $15 \mathrm{~min}$ ), the cells were collected, dissolved in $0.9 \% \mathrm{NaCl}$ and centrifuged again $(1000 \mathrm{~g}$ for $10 \mathrm{~min}$ at $4{ }^{\circ} \mathrm{C}$ ). Supernatant was drained and the cells were dissolved in RPMI 1640 medium with 10\% FCS and penicillin/streptomycin (cell final concentration was $10^{5} \mathrm{~mm}^{-3}$ ). The cells were allocated into six experimental groups, each containing six samples in a following way: the first group of hepatocytes was treated with anti-Fas antibodies in final concentration of $100 \mathrm{ng} \mathrm{ml} \mathrm{m}^{-1}$; the second group was treated with anti-Fas antibodies (in the same concentration) and $1 \mathrm{mM}$ melatonin; the third group was treated with anti-Fas antibodies (in the same concentration) and $1 \mathrm{mM}$ betaine hydrochloride; the fourth group was treated with melatonin only (the same concentration); the fifth group was treated with betaine hydrochloride only (in the same concentration); the sixth group was a control with standard culture conditions (RPMI 1640 medium, 10\% FCS and penicillin/streptomycin). The cells were grown for $24 \mathrm{~h}$, afterwards they were centrifuged $\left(1000 \mathrm{~g}\right.$ for $10 \mathrm{~min}$ at $\left.4^{\circ} \mathrm{C}\right)$, the 
supernatant was collected and the cells were resuspended in $1.2 \mathrm{ml}$ of a physiological saline solution.

\section{Detection of PS membrane externalization, oxidative stress generation, plasma and nuclear membrane integrity and nuclear fragmentation of isolated hepatocyte culture}

In order to detect PS on the membrane external leaflet, Annexin V was used. To the amount of $500 \mu \mathrm{l}$ of cells suspension, the Annexin V-FITC (Santa Cruz Biotechnology, USA) solution was added and fluorescence was detected on a flowcytometer (Epics-XL Coulter, Germany). Nuclear membrane permeability (usually seen in dead cells) was detected adding the equal aliquot of propidium iodide (PI) (Santa Cruz Biotechnology, USA) to cells suspension. Viable cells do not normally bind these chemicals. Annexin $\mathrm{V}$ positive, PI negative cells represent early apoptosis events. Both, Annexin V and PI positive cells document late apoptosis. If the cells do not bind Annexin V, but bind PI, they are in the necrotic phase. In this way, the following possibilities are: Annexin V-/PI- (healthy cells); Annexin V+/PI- (early apoptosis); Annexin V+/PI+ (late apoptosis); Annexin V-/PI+ (necrosis).

Xanthine oxidase (XO) activity was evaluated quantifying the formation of uric acid by using method modified and explained in our previous report ${ }^{32}$ with the calculated molar absorption coefficient of uric acid $\left(1.22 \times 10^{4} \mathrm{l} \mathrm{mol}^{-1} \mathrm{~cm}^{-1}\right)$. In brief, the reaction mixture containing hepatocytes, treated with anti-Fas antibodies, melatonin and/or betaine, and Tris/ $\mathrm{HCl}$ buffer was preincubated at $37^{\circ} \mathrm{C}$. The reaction was started by adding xanthine and, after incubation, stopped by adding perchloric acid. The enzyme activity was determined spectrophotometrically by measuring the absorption at $293 \mathrm{~nm}$ associated with uric acid formation. Xanthine oxidase activity was expressed as $\mathrm{U} \mathrm{g}^{-1}$ protein.

Generation of malondialdehyde (MDA) was used as an index of lipid peroxidation intensity in isolated hepatocytes, evaluated spectrophotometrically, according to the thiobarbituric acid (TBA) assay. ${ }^{33}$ The MDA level was expressed as nmol (MDA)/mg (proteins).

For detection of plasma membrane integrity and cell damage, the liberation of hepatic plasma membrane alkaline phosphatase (ALP) and gamma-glutamil transferase $(\gamma$-GT), and cellular enzymes alanine aminotransferase (ALT) and aspartate aminotransferase (AST) were all measured in collected supernatant using the Dade Behring RxL Max automatic analyzer. The activity of enzymes, evaluated quantifying the liberation in collected supernatant, was expressed as $\mathrm{U}^{-1}$.

The activity of alkaline DNase was measured spectrophotometrically at $260 \mathrm{~nm}$ by the method of Bartholeyns et al. ${ }^{34}$ modified in our laboratory for cell culture systems. ${ }^{5}$ The method is based on liberated acid soluble nucleotide determination, after precipitation, at $\mathrm{pH} 7.6$ obtained by adding Tris/HCl buffer and in the presence of $\mathrm{Mg}^{2+}$ ions as activators, using corresponding standard, commercial DNase I (Sigma Aldrich, USA). The enzyme activity was expressed as $\mathrm{U} \mathrm{g}^{-1}$ protein.

\section{Detection of Bcl-2 and Bax expression}

For determining the levels of Bcl-2 and Bax proteins, hepatocytes were isolated and treated as it was described in the previous section (Isolation of primary hepatocyte culture). Obtained cells were further processed according to the protocol by Kocic et al. 2011..$^{35}$ Briefly, the hepatocytes, resuspended in U-bottom 96-well culture plates, were fixed using $70 \%$ methanol and permeabilized with $0.1 \%$ Triton PBS. Specific antibodies were used: for Bcl-2 (N-19: sc-492 Santa Cruz Biotechnology, USA) and for Bax (P-19: sc-526 Santa Cruz Biotechnology, USA). The cells were incubated with the primary anti-Bcl-2 and antiBax, washed three times, and incubated with the FITCconjugated secondary antibodies. The excess of antibodies following staining was washed adding PBS (phosphate buffer saline) to each well. The mean fluorescence intensity (MFI; logarithmic scale) of cell populations was obtained following subtraction of blank values obtained by the treatment with secondary antibodies only.

\section{Nanoliposome encapsulation with melatonin or betaine hydrochloride and detection of susceptibility to $\mathrm{Fe}^{2+}$ and $\mathrm{H}_{2} \mathrm{O}_{2}$ induced oxidative stress}

In order to detect percentage of non-receptor phospholipid membrane melatonin uptake, phospholipid nanoparticles in the form of nanospheres, purchased from Nattermann Phospholipids (Germany) were dissolved as a $10 \%$ solution and one group was encapsulated by melatonin or by betaine hydrochloride at a concentration of $1 \mathrm{mM}$. Encapsulated nanoparticles were isolated by centrifugation at $6500 \mathrm{~g}$ for $30 \mathrm{~min}$ at $4{ }^{\circ} \mathrm{C}$ according to the method of Gortzi et al. ${ }^{36}$ In obtained supernatant melatonin concentration was estimated by using HPLC method ${ }^{37}$ and betaine hydrochloride by method of Storey and Jones ${ }^{38}$ and calculated as the encapsulation efficiency (\%), according to the corresponding standard of melatonin or betaine hydrochloride $(1 \mathrm{mM})$ dissolved in non-encapsulated liposome supernatant.

Sedimented nanoparticles were suspended in water and both encapsulated and native nanoparticles were exposed to oxidative stress by incubation with $\mathrm{H}_{2} \mathrm{O}_{2}$ or $\mathrm{FeSO}_{4}$ in a reaction mixture at a concentration of $1 \mathrm{mM}$ at $37^{\circ} \mathrm{C}$ for another $30 \mathrm{~min}$. The intensity of lipid peroxidation was estimated by the reaction of terminal lipid peroxidation product MDA with TBA, and measured spectrophotometrically at the wavelength of $532 \mathrm{~nm}$, according to the method of Buege and Aust. ${ }^{33}$ The MDA level was expressed as nmol (MDA)/ml (nanoliposome mixture).

\section{In silico study of physico-chemical properties of melatonin and betaine}

The physico-chemical properties of melatonin and betaine, such as lipophilicity (octanol-water partition coefficient and polar surface area), molecular size (molecular weight and volume), conformational flexibility (number of rotatable bonds) and number of present hydrogen-donors and acceptors, were calculated by using in silico Molinspiration tool. ${ }^{30} \mathrm{With}$ physicochemical characteristics taken into account, some other important features, relating to the behavior and interaction of compounds to the membrane, were predicted. ACD/I-Lab software $^{31}$ was used in order to estimate the membrane permeability of both tested compounds through the quantified 
percentage represented information about their absorption rate and bioavailability predicted on the basis of their chemical structure.

\section{Statistical analysis}

The results are presented as mean \pm SD. The statistical significance was evaluated using ANOVA test. A $p$-value of 0.05 was considered statistically significant.

\section{Results}

\section{Detection of PS membrane externalization, oxidative stress generation, plasma and nuclear membrane integrity and nuclear fragmentation of isolated hepatocyte culture}

Fig. 1 illustrates PS membrane externalization of mouse hepatocytes assayed by Annexin V/PI tests. The treatment of hepatocytes with anti-Fas antibodies significantly increased the number of Annexin V+ hepatocytes, where PS was on the external membrane leaflet. Melatonin greatly preserved the number of Annexin V-/PI- cells, when was co-incubated with anti-Fas antibodies, compared to only anti-Fas antibodies treated hepatocytes. Contrary to melatonin, betaine hydrochloride was able to achieve protective effect neither alone nor in co-incubation with anti-Fas antibodies. According to obtained results, it is supposed that in the early phase of apoptosis melatonin exerts antioxidative effect in order to reduce the generation of free-radicals and subsequent oxidation and externalization of PS. Only antioxidative effect was shown as non sufficient for maintaining the membrane stability. A more effective protective effect of melatonin was exerted in the late apoptotic conditions, when antioxidative effects were manifested in a synergy with antiapoptotic effects latter discussed. Betaine exerted even stronger effects on nuclear membrane disintegration, regarding the quantity of Annexin V+/PI+ cells. Melatonin combined with anti-Fas antibodies significantly decreased the quantity of late apoptotic Annexin V+/PI+ cells. Betaine hydrochloride alone increased the level of Annexin V+/PI+ cells compared to control hepatocytes. There were no Annexin $\mathrm{V}-/ \mathrm{PI}+$ cells which were considered necrotic.

The obtained results provided evidence that melatonin exerts hepatoprotective effects especially in the late phases of apoptosis, by maintaining plasma and nuclear membrane integrity. Betaine hydrochloride would not be considered as protector of membrane stability, it exerted strong effect on nuclear membrane disintegration.

As shown in Fig. 2, exposure to anti-Fas antibodies and their combination with betaine hydrochloride induced an increase in lipid peroxidation (LP), when compared to the control hepatocytes. Decrease in LP was evidenced in the combined treatment with anti-Fas antibodies and melatonin in comparison with only anti-Fas antibodies-treated hepatocytes or the combination of anti-Fas antibodies and betaine-treated hepatocytes. Melatonin, given alone, decreased the intensity of LP in comparison with control hepatocytes. Betaine hydrochloride given alone was without significant effect. Melatonin as antioxidant prevented the peroxidation of membrane lipids in mouse hepatocytes and maintained membrane stability, while betaine hydrochloride could not be considered as hepatoprotective agent.

As depicted in Fig. 3, exposure to anti-Fas antibodies induced an increase in xanthine oxidase (XO) activity, when compared to the control hepatocytes. Significant decrease in XO

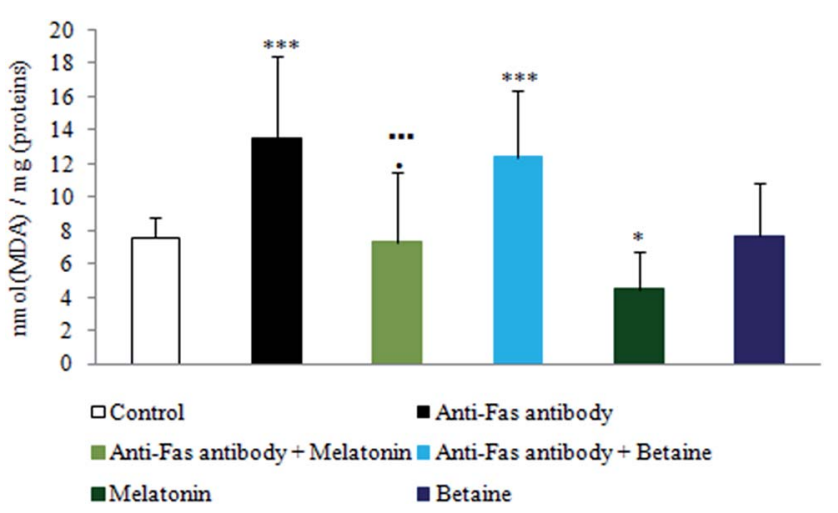

Fig. 2 The intensity of lipid peroxidation in hepatocytes treated with anti-Fas antibodies, melatonin and betaine. Generation of malondialdehyde (MDA) used as an index of lipid peroxidation intensity was evaluated spectrophotometrically and expressed as nmol (MDA)/mg (proteins). ${ }^{* * *} p<0.001$ vs. control; $* p<0.05$ vs. control; $\mathbf{m} \mathbf{m} p<$ 0.001 anti-Fas antibody vs. anti-Fas antibody + melatonin; $p<0.05$ anti-Fas antibody + melatonin vs. anti-Fas antibody + betaine.
A

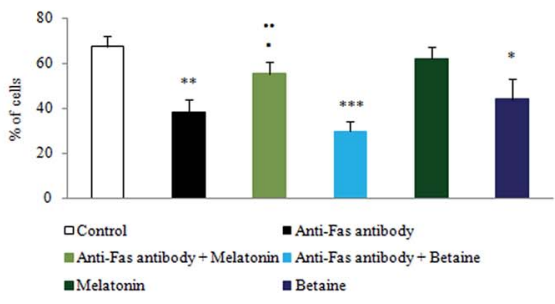

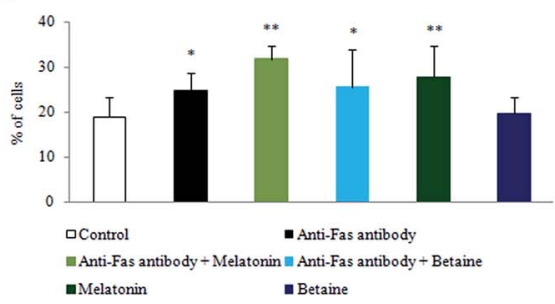

C

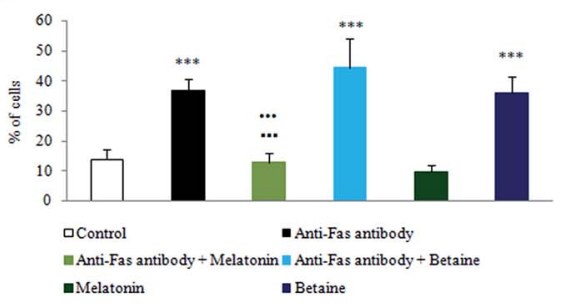

Fig. 1 PS membrane externalization and changes in nuclear membrane integrity of mouse hepatocytes treated with anti-Fas antibodies, melatonin and betaine, assayed by Annexin V/PI tests. The represented stages are: (A) Annexin V-/PI- (healthy cells); (B) Annexin V+/PI- (early apoptosis); (C) Annexin V+/PI+ (late apoptosis). $* * * p<0.001$ vs. control; **p< 0.01 vs. control; $* p<0.05$ vs. control; $\mathbf{E}$. $p<0.001$ anti-Fas antibody vs. anti-Fas antibody + melatonin; $p<0.05$ anti-Fas antibody vs. anti-Fas antibody + melatonin; $\bullet \bullet \bullet p<0.001$ anti-Fas antibody + melatonin vs. anti-Fas antibody + betaine; $\bullet \bullet p 0.01$ anti-Fas antibody + melatonin vs. anti-Fas antibody + betaine. 
activity was evidenced in the combined treatment with anti-Fas antibodies and melatonin in comparison with only anti-Fas antibodies treated hepatocytes. Melatonin, given alone, decreased the activity of this enzyme in the comparison with

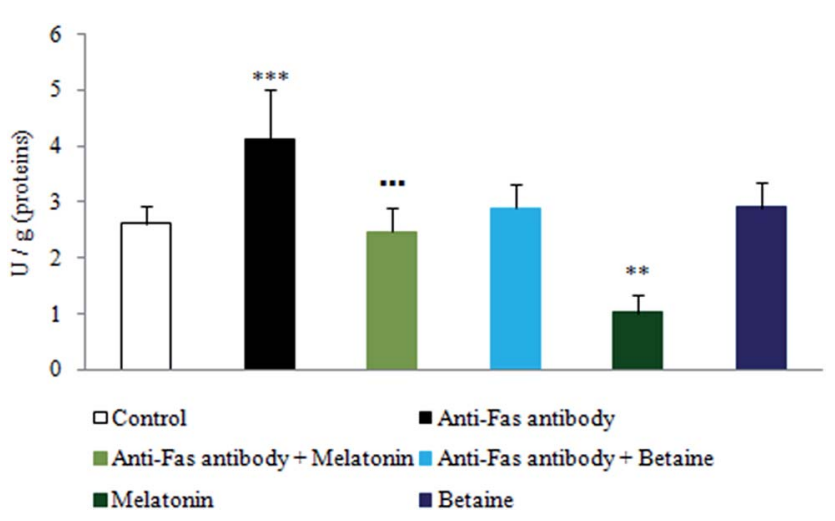

Fig. 3 Xanthine oxidase activity in hepatocytes treated with anti-Fas antibodies, melatonin and betaine. The enzyme activity, evaluated spectrophotometrically quantifying the formation of uric acid, was expressed as $\cup \mathrm{g}^{-1}$ protein. ${ }^{* * *} p<0.001$ vs. control; $* * p<0.01$ vs. control; $\mathbf{E}_{p}<0.001$ anti-Fas antibody vs. anti-Fas antibody + melatonin.

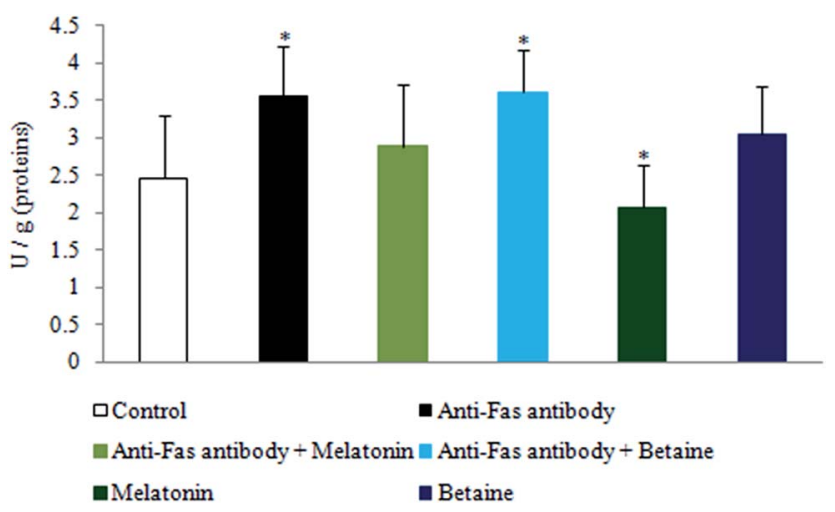

Fig. 4 DNase activity in hepatocytes treated with anti-Fas antibodies, melatonin and betaine. The activity of alkaline DNase was spectrophotometrically measured by the liberation of acid-soluble nucleotides and expressed as $\mathrm{U} \mathrm{g}^{-1}$ protein. ${ }^{*} p<0.05$ vs. control. control hepatocytes. Betaine hydrochloride given alone was without significant effect. In that way, melatonin decreased the generation of free-radicals by the XO catalyzed reactions, what represents one more mechanism of its indirect antioxidative effect. Betaine was without significant effect on the activity of this enzyme.

The exposure to anti-Fas antibodies, specifically their combination with betaine hydrochloride, induced an increase in DNase activity when compared to the control hepatocytes. Melatonin, given alone, decreased the DNase activity in the comparison with control hepatocytes. Betaine hydrochloride given alone was without significant effect (Fig. 4). Besides the protection of DNA from oxidative damage, melatonin may protect it by the inhibition of DNase activity as well. Destabilization of DNA potentially may be provoked by betaine.

The integrity of hepatocytes and their damage was assessed by the levels of liberated enzymes due to cell damage. The hepatocytes treated with anti-Fas antibodies were evidenced by significant increase $(p<0.001)$ in the liberation of AST, ALT, ALP and $\gamma$-GT when compared to control cell supernatant (Table 1). However, melatonin exhibited hepatoprotective effects and attenuated the elevated activity of liberated hepatocyte enzymes, with significant effectiveness when was combined with anti-Fas antibodies in comparison to alone anti-Fas antibody treatment $(p<0.001)$. Betaine exerted a less effective hepatoprotective effect.

\section{Detection of Bcl-2 and Bax expression}

Fig. 5 shows Bcl-2 and Bax protein expression on the mitochondrial membrane under the given experimental conditions. The treatment with anti-Fas antibodies led to inverse relation between Bcl-2 and Bax protein compared to control hepatocytes, reducing the values of Bcl-2 and enhancing Bax membrane expression. Melatonin almost fully preserved mitochondrial Bcl-2/Bax expression and distribution, while betaine hydrochloride was without significant effect under the co-incubation conditions with anti-Fas antibodies, compared to anti-Fas antibodies treated hepatocytes. Melatonin, given alone, significantly attenuated Bax expression in the cell culture, compared to control cells, while betaine hydrochloride alone significantly decreased Bcl-2 expression, compared to the control hepatocytes. Thus, melatonin was

Table 1 The integrity of hepatocytes treated with anti-Fas antibodies, melatonin and betaine assessed by the levels of liberated enzymes ${ }^{a}$

Experimental groups of

\begin{tabular}{|c|c|c|c|c|}
\hline hepatocytes/enzymes $\left(\mathrm{U} \mathrm{l}^{-1}\right)$ & AST & ALT & $\gamma$-GT & ALP \\
\hline Anti-Fas antibody & $166.15 \pm 23.45^{* * *} \square \square$ & $616.68 \pm 83.85^{* * *} \square \square$ & $7.52 \pm 1.95 * * * \square$ & $27.85 \pm 5.23 * * * \square=$ \\
\hline Anti-Fas + betaine & $98.00 \pm 12.54^{* * *}$ & $459.12 \pm 62.34^{* * *}$ & $7.00 \pm 1.34 * * * \bullet$ & $13.60 \pm 3.87$ \\
\hline Melatonin & $50.25 \pm 12.22$ & $329.75 \pm 42.81$ & $3.25 \pm 1.02^{* * *}$ & $8.75 \pm 2.97 * * *$ \\
\hline Betaine & $86.50 \pm 33.34^{* * *}$ & $324.71 \pm 53.13$ & $6.50 \pm 2.95$ & $10.50 \pm 3.19$ \\
\hline
\end{tabular}

$86.50 \pm 33.34^{* * *}$

${ }^{a}$ The activities of liberated aspartate aminotransferase (AST), alanine aminotransferase (ALT), alkaline phosphatase (ALP) and gamma-glutamil transferase $\left(\gamma\right.$-GT) were measured in collected supernatant and expressed as $\mathrm{U}^{-1}$. $* * * p 0.001 v s$. control; $\mathbf{\square} \mathbf{\square}<0.001$ anti-Fas antibody $v$ s. anti-Fas antibody + melatonin; $\boldsymbol{\bullet}_{p}<0.05$ anti-Fas antibody $v s$. anti-Fas antibody + melatonin; $\bullet \bullet \bullet p<0.001$ anti-Fas antibody + melatonin $v s$. anti-Fas antibody + betaine; $\bullet<0.05$ anti-Fas antibody + melatonin $v s$. anti-Fas antibody + betaine. 
A

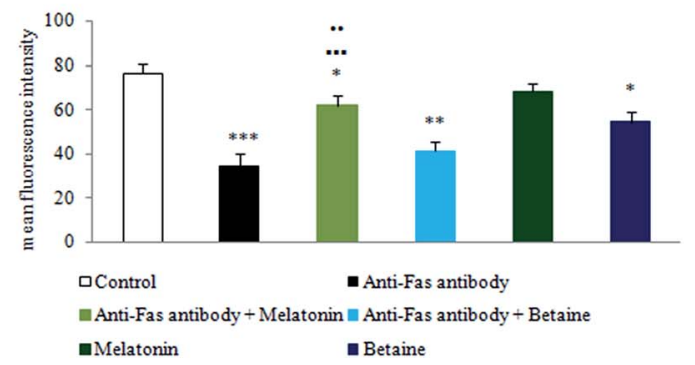

B

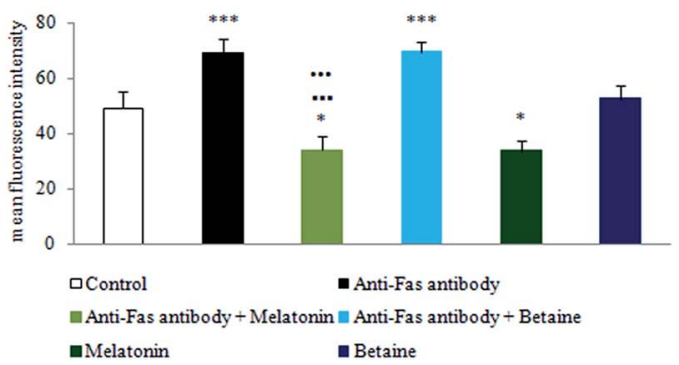

Fig. $5 \mathrm{Bcl}-2(\mathrm{~A})$ and $\mathrm{Bax}(\mathrm{B})$ expression in hepatocytes treated with anti-Fas antibodies, melatonin and betaine. The mean fluorescence intensity of cells indicated for both, up and down regulation of populations for compounds and antibodies used, compared to intact hepatocytes. *** $p<$ 0.001 vs. control; ${ }^{* *} p<0.01$ vs. control; $* p<0.05$ vs. control; $\boldsymbol{m}^{*} p<0.001$ anti-Fas antibody vs. anti-Fas antibody + melatonin; $\bullet \bullet \bullet p<0.001$ anti-Fas antibody + melatonin vs. anti-Fas antibody + betaine; $\bullet \bullet p<0.01$ anti-Fas antibody + melatonin vs. anti-Fas antibody + betaine.

shown to increase the expression of $\mathrm{Bcl}-2$ and to decrease the expression of Bax protein in mouse hepatocytes, indicating decreased susceptibility of these cells to apoptosis and hepatic damage. Betaine hydrochloride exerted the opposite effect.

\section{Melatonin and betaine hydrochloride nanoliposome encapsulation and detection of susceptibility to $\mathrm{Fe}^{2+}$ and $\mathrm{H}_{2} \mathrm{O}_{2}$ induced oxidative stress}

Results obtained by using nanoliposomes as a possible artificial membrane-like phospholipid system and potential drug

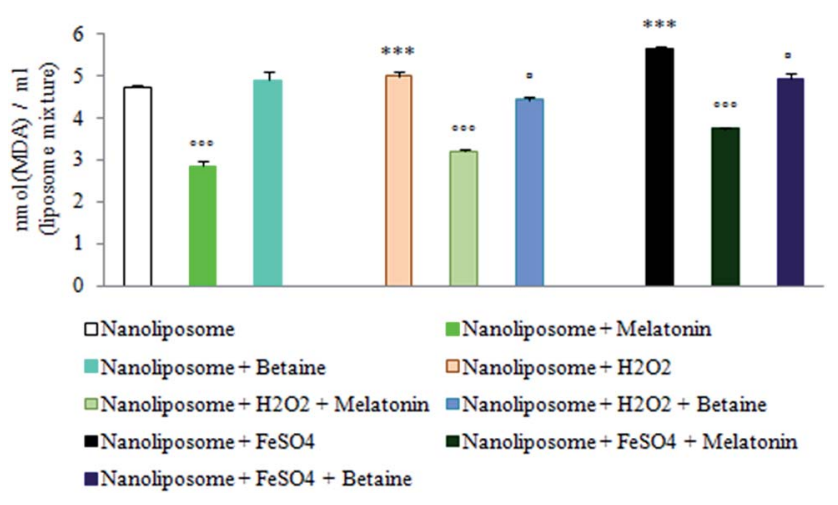

Fig. 6 Capability of melatonin- or betaine-encapsulated nanoliposome spheres to counteract $\mathrm{H}_{2} \mathrm{O}_{2}$ and $\mathrm{FeSO}_{4}$ induced oxidative stress. The intensity of lipid peroxidation was estimated by the reaction of MDA with TBA, and measured spectrophotometrically. The MDA level was expressed as nmol (MDA)/ml (nanoliposome mixture). $* * * p<$ 0.001 vs. nanoliposome; ${ }^{\cdots p} p 0.001$ melatonin-encapsulated nanoliposome vs. nanoliposome; $\square p<0.05$ betaine-encapsulated nanoliposome vs. nanoliposome. delivery system suggest that melatonin as a lipophilic molecule may freely cross external cell membrane and be localized within the lipid nanospheres in about $74.48 \pm 1.04 \%$, while the encapsulation efficiency of betaine hydrochloride was $51.40 \pm$ $3.99 \%$. In this way melatonin may additionally contribute to the intracellular membrane structure stability and counteract oxidative stress-induced membrane damage.

Fig. 6 shows the intensity of $\mathrm{H}_{2} \mathrm{O}_{2}$ and $\mathrm{FeSO}_{4}$ induced lipid peroxidation measured via thiobarbituric acid reactive substances (TBARS) production, regardless of the absence or presence of encapsulated melatonin or betaine hydrochloride in phospholipid liposome nanospheres. According to the results of the encapsulation of nanoliposomes with betaine hydrochloride, it would not be considered as a successful drugdelivery system. $\mathrm{Fe}^{2+}$-dependent TBARS (MDA) production in nanoliposomes was markedly enhanced compared to $\mathrm{H}_{2} \mathrm{O}_{2}$, significantly suppressed after melatonin encapsulation, but not after betaine hydrochloride encapsulation.

\section{In silico study of calculated molecular physico-chemical properties of melatonin and betaine}

Table 2 represents molecular physico-chemical properties of melatonin and betaine calculated by using Molinspiration tool. ${ }^{30}$ Lipinski and coworkers established an effective methodology for estimation of potential drug solubility and permeability based on the calculation of molecular weight, octanol/ water partition coefficient, number of $\mathrm{H}$-bond donors and acceptors. Based on the analysis of large number of drugs, a cutoff for each of the four physico-chemical properties was set into the "Rule of five": poor absorption or permeation are more likely to occur when the molecule has molecular weight more

Table 2 Molecular physico-chemical properties of melatonin and betaine calculated by using in silico Molinspiration tool ${ }^{30}$

\begin{tabular}{|c|c|c|c|c|c|c|c|c|c|}
\hline Compound & $m_{\mathrm{i}} \log P^{a}$ & TPSA $^{b}$ & $N_{\text {atoms }}{ }^{c}$ & $\mathrm{MW}^{d}$ & $N_{\mathrm{ON}}{ }^{e}$ & $N_{\text {OHNH }}{ }^{f}$ & $N_{\text {viol. }}^{g}{ }^{g}$ & $N_{\text {rotb. }}{ }^{h}$ & $\mathrm{Vol}^{i}$ \\
\hline Melatonin & 1.45 & 54.12 & 17 & 232.28 & 4 & 2 & 0 & 4 & 220.12 \\
\hline Betaine & -5.41 & 40.13 & 8 & 117.15 & 3 & 0 & 0 & 2 & 119.60 \\
\hline
\end{tabular}

${ }^{a}$ Octanol-water partition coefficient, calculated by the methodology developed by Molinspiration. ${ }^{b}$ Polar surface area. ${ }^{c}$ Number of nonhydrogen atoms. ${ }^{d}$ Molecular weight. ${ }^{e}$ Number of hydrogen-bond acceptors ( $\mathrm{O}$ and $\mathrm{N}$ atoms). ${ }^{f}$ Number of hydrogen-bond donors (OH and NH groups).

${ }^{g}$ Number of "Rule of five" violations. ${ }^{h}$ Number of rotatable bonds. ${ }^{i}$ Molecular volume. 
than 500, $\log P$ over 5 , and contains more than $5 \mathrm{H}$-bond donors or $10 \mathrm{H}$-bond acceptors. More than one violation of the rule is the critical limit for acceptable drug-likeness. ${ }^{39}$ Summarizing the calculated physico-chemical properties of melatonin and betaine, it was concluded that both compounds obey the Lipinski "Rule of five" and meet all criteria for good solubility and permeability. Based on the physico-chemical determinants, absorption rate and oral bioavailability were predicted in order to gain a better insight into membrane permeability for melatonin and betaine. According to the results obtained by using $\mathrm{ACD} / \mathrm{I}-\mathrm{Lab}$ software ${ }^{31}$ betaine was predicted to have poor passive absorption of $34 \%$ (paracellular route), while melatonin was shown to have complete passive absorption of $100 \%$ (transcellular route).

\section{Discussion}

Membrane phospholipids represent a specific sensing system for external "death signals" or for intracellular damage, undergoing to perturbations of the membrane lipid bilayer in a way that phosphatidylserine (PS) is redistributed from the inner to the outer leaflet of the plasma membrane. The appearance of anionic PS at the cell surface is the most remarkable feature in apoptosis because of the abundance of this phospholipid, its negative charge and the ability to change interactions with other lipids that probably disrupts lipid rafts. ${ }^{40}$ There is accumulating evidence showing that the generation of reactive oxygen species and subsequent oxidative stress associated with the initiation of apoptosis leads to selective oxidation of phospholipids such as PS in the cytoplasmic layer of membrane, followed by egress of oxidized PS (oxPS) to the surface of the apoptotic cells, effective recognition and cell clearance by phagocytes. ${ }^{41,42}$

It has been shown that Fas-mediated apoptosis induces caspase-3 activation, chromatin condensation, PS externalization, generation of reactive oxygen species, intracellular glutathione depletion, disruption of mitochondrial transmembrane potential, release of cytochrome c (cyt c) from mitochondria, followed by the increased presence of oxPS within the cells and on the cell surface. ${ }^{42}$ Mitochondria play an essential role in production of oxPS and its subsequent transmigration to the cell surface during extrinsic apoptosis. ${ }^{43}$ Serving as a naturally mitochondria-targeted protective molecule, ${ }^{18}$ melatonin regulates membrane stability. ${ }^{7}$ It is considered that a potential mechanism underlying selective oxidation of PS by $\mathrm{H}_{2} \mathrm{O}_{2}$ during apoptosis relies on the specific interactions between positively charged cyt $\mathrm{c}$ and negatively charged PS. Formed oxPS (PSOOH) may function as a substrate for cyt c peroxidase activity and further propagate lipid peroxidation. Therefore, the departure of cyt $\mathrm{c}$ from mitochondria is required for production of oxPS as well as its externalization. It has been reported that oxPS facilitates translocation of both PS and oxPS molecules to the cell surface. ${ }^{41}$ By the decrease of the Bax expression reported in our study, melatonin may inhibit the release of cyt c from mitochondria and, additionally, in this way, besides its antioxidative activity, reduce PS externalization.
Moreover, it has been shown that the inhibition of caspase 8 reduces mitochondrial cyt $\mathrm{c}$ release, the amount of oxPS and caspase 3 activation in Fas-induced apoptosis. ${ }^{\mathbf{1} 41}$ Suppression of caspase-8 activity in Fas-mediated apoptotic pathways provides a new insight into the cytoprotective effect of melatonin. ${ }^{\mathbf{4}}$ It has been found that melatonin abrogates the caspase8 activity in rabbit liver modulating TNF-mediated apoptotic pathway. ${ }^{45}$ On the other hand, cyt c release induced by the proapoptotic Bcl-2 family member Bid inserted in the outer mitochondrial membrane can be inhibited by Bcl-2 overexpression. The overexpression of $\mathrm{Bcl}-2$ protects against Fas-mediated apoptosis. ${ }^{1}$ By the increase of Bcl-2 expression reported in this study, melatonin may inhibit mitochondrial release of cyt c, as well. The apoptotic-induced production, and externalization of oxPS has been detected in patients with alcoholic liver disease. ${ }^{46}$

In the study on prevention of bile acid-induced apoptosis by betaine in rat hepatocytes in vitro and in vivo, it has been shown that betaine affected neither the bile acid-induced membrane trafficking of CD95 and tumor necrosis factor receptor nor recruitment of Fas-associated death domain and caspase 8 to the CD95 receptor. ${ }^{47}$ By using a model of Fas ligand-induced apoptosis, we have obtained similar results.

Understanding the interaction and positioning of melatonin in biological membranes is an essential step towards decoding the mechanism of its influence on the membrane integrity, its protective abilities ${ }^{\mathbf{4 8}}$ and potential delivery of nanoliposome. Results of the evaluation of non-receptor phospholipid membrane melatonin uptake indicated that more than $74 \%$ of melatonin was encapsulated into lipid nanospheres, where it was able to counteract oxidative stress-induced membrane damage (Fig. 6). Since lipid peroxidation of cell membrane phospholipids caused by free-radicals has been proposed to be a major mechanism for the onset of a number of pathological events including apoptosis, the obtained result may suggest that membrane localization of melatonin may serve as the first line of defense against cell damage and apoptosis, which would not be considered in the case of betaine hydrochloride.

These results are in accordance with other literature data. ${ }^{47-51}$ Assuming that melatonin is located in the proximity of the polar heads of membrane phospholipids, molecules should exaggerate its activity against free-radicals coming from the aqueous compartment $^{50}$ protecting phospholipids from oxidation. Understanding the organization and interactions of melatonin in membranes may shed light into its antioxidative and cytoprotective properties, ${ }^{48}$ and help develop a structural basis for construction of encapsulated nanoliposomes as suitable drug delivery system. By virtue of its ability to protect lipids from freeradical damage, melatonin is remarkably beneficial in preserving the morphological and functional integrity of cell membranes, in maintaining them at optimal fluidity and preventing them from becoming rigid. This contributes significantly to the function of proteins (receptors, channels, pores, etc.) in the cell membranes and helps in preserving their functional stability. ${ }^{17}$

The change in the $\mathrm{Bax} / \mathrm{Bcl}-2$ ratio may cause mitochondrial damage mediated by the breakdown of the mitochondrial transmembrane potential and the release of cyt $\mathrm{c}$ into the 
cytoplasm along with subsequent caspase activation in the final apoptotic phenotype. The relative expression of antiapoptotic $\mathrm{Bcl}-2$ is the key deciding point in regard to cell fate. ${ }^{20}$ Oxidative stress is considered to be an important mechanism in the apoptosis of hepatocytes and the resulting liver damage. Melatonin has been documented to play a direct role in mitochondrial homeostasis by scavenging reactive oxygen species (ROS) and reactive nitrogen species (RNS), improving mitochondrial membrane integrity. ${ }^{52}$ It has been shown that melatonin may significantly decrease the $\mathrm{Bax} / \mathrm{Bcl}-2$ ratio in irradiation-induced apoptosis in rats' peripheral blood lymphocytes and liver. ${ }^{20,53}$ Some BH3-only proteins such as Bim may directly bind to and activate Bax, which leads to its oligomerisation, the permeabilization of the mitochondrial outer membrane, cyt $c$ release and the activation of caspase cascade. ${ }^{54,55}$ Bim phosphorylation by the extracellular signal regulated kinase $1 / 2$ (ERK1/2) promotes its degradation through the proteasomeubiquitination system..$^{55}$ Contrary to Bim, the phosphorylation of Bcl-2 mediated by ERK2 might be of substantial importance for Bcl-2 stability and cell survival. Bcl-2 phosphorylation lends it its stability and rescues it from degradation and eventual cell death through the proteasome-ubiquitination system. ${ }^{56}$ The activity of ERK was shown to be significantly stimulated by melatonin, suggesting that the mitogen-activated protein kinase (MAPK)/ERK pathway is involved in constitutive and stress-induced signaling of melatonin to promote mitochondrial protection and cell survival. ${ }^{57}$ Moreover, it has been shown that the activation of the ERK1/2 pathway confers hepatocyte resistance to death. ${ }^{58}$ It has been shown that melatonin may sustain the activation of Akt. ${ }^{59-62}$ In that way, by the activation of Akt, melatonin may inhibit Bax proapoptotic actions. It has been documented that Akt protects mouse hepatocytes from TNF- $\alpha$ and Fas-mediated apoptosis. ${ }^{63}$ Therefore, this is one more possible mechanism of melatonin hepatoprotection mediated by the activation of Akt. Furthermore, $\mathrm{MT}_{1} / \mathrm{MT}_{2}$ signal transduction seems to be responsible for Bcl-2 re-localization to mitochondria, suggesting that Bax activation may in fact be antagonized by Bcl-2 at the mitochondrial level. ${ }^{64}$ Bax translocation to the mitochondria and the antiapoptotic effect of melatonin have been shown to be dependent on the presence of Bcl-2 re-localized in the mitochondria. ${ }^{65}$

Changes in the mitochondrial membrane can trigger further DNA cleavage, including nuclear membrane disintegration. ${ }^{2}$ Melatonin was observed to be a protector of nuclear DNA fragmentation. The possible mechanisms would be free-radicals scavenging property, significant inhibition of free-radicals' formation by xanthine oxidase (Fig. 3) and the inhibition of DNase activity (Fig. 4). Obtained results are in accordance with those in our previous study about the protective effect of melatonin on rats exposed to microwave irradiation. ${ }^{\mathbf{1 3 - 1 5}}$ In the present study, it was shown that exposure to betaine in Fasmediated apoptotic conditions may induce an increase in DNase activity in treated hepatocytes. This result is in accordance with known literature data which claim that betaine was proven to considerably destabilize DNA. ${ }^{66}$

Liver biomarkers (AST, ALT, ALP and $\gamma$-GT) are important criteria for the evaluation of the structural integrity of hepatocytes and hepatotoxicity. The liberated amounts of enzymes indicate the severity of hepatocyte damage. In the present study, melatonin exhibited hepatoprotective effects by preserving liberation of hepatocyte enzymes, with significant effectiveness when it was combined with anti-Fas antibodies in comparison to anti-Fas antibody treatment alone. These results are in accordance with the literature data. ${ }^{67,68}$ Betaine combined with anti-Fas antibodies show less effectiveness in preserving membrane stability and liberation of hepatocyte enzymes. In the evaluation of the prevention of bile acid-induced apoptosis by betaine in rat hepatocytes in vitro and in vivo it has been shown that betaine may decrease the activity of these liver enzymes, but only to a low degree of effectiveness, when it was supplemented after bile duct ligation. ${ }^{47}$ Our results documented that betaine hydrochloride could not be considered as a protector against membrane PS externalization nor Bcl-2/Bax expression and redistribution, lipid peroxidation, generation of free-radicals in reactions catalyzed by xanthine oxidase and DNA fragmentation during apoptosis, when it was co-incubated with anti-Fas antibodies or given alone. Obtained results may be interpreted in terms of betaine structure and its transport protein dependence on surrounding membrane phospholipids. The membrane receptors and transport proteins function in a fluid lipid bilayer environment, which is crucially involved in their proper organization and function. The activity and regulation of lipid-associated BetP is strongly dependent on the presence of negatively charged lipids around it. ${ }^{26}$ These lipids may contribute to inactivation or activation of BetP via specific electrostatically favorable interactions. Modulation of transport protein activity by external stimuli may be an important aspect of cellular stress, including apoptosis. The influence of the membrane surface charge has shown that the increase of strongly negatively charged phospholipids may shift the threshold of activation or may lead to inactive BetP conformation. ${ }^{69}$

The lipophilicity of compounds is a common property used to estimate the membrane permeability of a molecule. It is often measured as the $\log$ of the partition coefficient between $n$ octanol and water. The partition coefficient values (Table 2), calculated by using the Molinspiration tool, ${ }^{30}$ indicated that betaine is more hydrophilic than melatonin, which means that it may show lower membrane permeation, absorption rate and bioavailability. Regarding hydrogen binding capacity, both melatonin and betaine possess less than $5 \mathrm{H}$-bond donors and acceptors. The conformational flexibility of the molecules described by the number of rotatable bonds is important factor, among others, for the assessment of the binding efficiency of receptors and channels. The satisfactory number of rotatable bonds is 10 or fewer, and both of the tested compounds meet these criteria. In addition to the number of rotatable bonds, Veber and coworkers found that the polar surface area can be used as a good indication of permeation. The membrane permeation rate is recognized as a common requirement for bioavailability. The higher hydrophilicity makes the interaction and uptake over a lipid bilayer difficult. The suggested Veber rules are: acceptable oral bioavailability is more likely to occur when the molecule has 10 or fewer rotatable bonds, and polar surface area equal to or less than $140 \AA^{2}$ and 12 or fewer 
H-donors and acceptors. Veber and coworkers therefore also raise the issue of molecular weight as a proper descriptor for permeation and absorption measurement, as it might just be positively correlated with more precise properties like the rotatable hydrogen bonds count and polar surface area. ${ }^{70}$ It was concluded that both melatonin and betaine obey the Veber rules. The data obtained by $\mathrm{ACD} / \mathrm{I}-\mathrm{Lab}^{31}$ indicate that the oral bioavailability of melatonin is more than $70 \%$, and of betaine less than $30 \%$. These values are in accordance with criteria of Egan rule, relating to the assessment of potential drug oral bioavailability based on the calculation of the octanol/water partition coefficient and polar surface area. ${ }^{71}$ All of the above may indicate that the cell membrane permeation of melatonin is higher than the permeation of betaine.

In conclusion, increased formation of reactive oxygen species and lipid peroxidation in anti-Fas-antibody induced apoptosis was reduced by melatonin. In this way, melatonin may contribute to mitochondrial membrane stability and integrity and to nuclear membrane stability. The above mentioned effects are supported by data on the physicochemical characteristics of both tested compounds obtained using in silico studies. Considering the importance of apoptosis in a variety of liver diseases, the evidence and clarified mechanisms of melatonin actions in the apoptotic and oxidative processes, knowing its physico-chemical properties, represent important prerequisites for the formulation of future non-toxic nanoliposome-mediated therapy for clinical practice. In order to provide recommendations for successful treatment of hepatic apoptosis, it could therefore be preferable the use of lipophylic compounds, like melatonin, rather than more hydrophilic ones like betaine.

\section{Acknowledgements}

The study was supported by the projects TR 31060 and OI 172044 of Ministry of Education and Science of the Republic of Serbia. Voja Pavlovic was carried Annexin/PI test.

\section{References}

1 M. L. Bajt, J. A. Lawson, S. L. Vonderfecht, J. S. Gujral and H. Jaeschke, Protection against Fas receptor-mediated apoptosis in hepatocytes and nonparenchymal cells by a caspase-8 inhibitor in vivo: evidence for a postmitochondrial processing of caspase-8, Toxicol. Sci., 2000, 58(1), 109-117.

2 W. O. Pereira and G. P. Amarante-Mendes, Apoptosis: a programme of cell death or cell disposal?, Scand. J. Immunol., 2011, 73(5), 401-407.

3 V. Depraetere and P. Golstein, Fas and other cell death signaling pathways, Semin. Immunol., 1997, 9(2), 93-107.

4 K. Higaki, H. Yano and M. Kojiro, Fas antigen expression and its relationship with apoptosis in human hepatocellular carcinoma and noncancerous tissues, Am. J. Pathol., 1996, 149(2), 429-437.

5 G. Kocic, G. Bjelakovic, D. Pavlovic, T. Jevtovic, V. Pavlovic, D. Sokolovic, et al., Protective effect of interferon-alpha on the DNA and RNA-degrading pathway in anti-Fas-antibody induced apoptosis, Hepatol. Res., 2007, 37(8), 637-646.

6 A. Shiratsuchi, S. Osada, S. Kanazawa and Y. Nakanishi, Essential role of phosphatidylserine externalization in apoptosing cell phagocytosis by macrophages, Biochem. Biophys. Res. Commun., 1998, 246(2), 549-555.

7 J. León, D. Acuña-Castroviejo, G. Escames, D. X. Tan and R. J. Reiter, Melatonin mitigates mitochondrial malfunction, J. Pineal Res., 2005, 38(1), 1-9.

8 B. Antonsson and J. C. Martinou, The Bcl-2 protein family, Exp. Cell Res., 2000, 256, 50-57.

9 P. Guo, H. Pi, S. Xu, L. Zhang, Y. Li, M. Li, et al., Melatonin improves mitochondrial function by promoting MT1/ SIRT1/PGC-1 alpha-dependent mitochondrial biogenesis in cadmium-induced hepatotoxicity in vitro, Toxicol. Sci., 2014, 142(1), 182-195.

10 C. von Gall, J. H. Stehle and D. R. Weaver, Mammalian melatonin receptors: molecular biology and signal transduction, Cell Tissue Res., 2002, 309(1), 151-162.

11 D. X. Tan, L. C. Manchester, M. P. Terron, L. J. Flores and R. J. Reiter, One molecule, many derivatives: a never ending interaction of melatonin with reactive oxygen and nitrogen species?, J. Pineal Res., 2007, 42(1), 28-42.

12 R. J. Reiter, D. X. Tan, L. C. Manchester and W. Qi, Biochemical reactivity of melatonin with reactive oxygen and nitrogen species: a review of the evidence, Cell Biochem. Biophys., 2001, 34(2), 237-256.

13 D. Sokolovic, B. Djindjic, J. Nikolic, G. Bjelakovic, D. Pavlovic, G. Kocic, et al., Melatonin reduces oxidative stress induced by chronic exposure of microwave radiation from mobile phones in rat brain, J. Radiat. Res., 2008, 49(6), 579-586.

14 D. Sokolovic, B. Djordjevic, G. Kocic, A. Veljkovic, M. Marinkovic, J. Basic, et al., Melatonin protects rat thymus against oxidative stress caused by exposure to microwaves and modulates proliferation/apoptosis of thymocytes, Gen. Physiol. Biophys., 2013, 32(1), 79-90.

15 B. Djordjevic, D. Sokolovic, G. Kocic, A. Veljkovic, M. Despotovic, J. Basic, et al., The effect of melatonin on the liver of rats exposed to microwave radiation, Bratisl. Lek. Listy, 2015, 116(2), 96-100.

16 V. N. Anisimov, I. G. Popovich, M. A. Zabezhinski, S. V. Anisimov, G. M. Vesnushkin and I. A. Vinogradova, Melatonin as antioxidant, geroprotector and anticarcinogen, Biochim. Biophys. Acta, 2006, 1757, 573-589.

17 R. J. Reiter, L. Fuentes-Broto, S. D. Paredes, D. X. Tan and J. J. Garcia, Melatonin and the pathophysiology of cellular membranes, Marmara Pharm. J., 2010, 14(1), 1-9.

18 O. Celik and M. Naziroğlu, Melatonin modulates apoptosis and TRPM2 channels in transfected cells activated by oxidative stress, Physiol. Behav., 2012, 107, 458-465.

19 E. Issabeagloo, F. Ahmadpoor, P. Kermanizadeh and M. Taghizadiyeh, Hepstoprotective actions of melatonin against methotrexate induced hepatic injury in animal model, Curr. Res. J. Biol. Sci., 2011, 6(8), 381-385.

20 M. Mohseni, E. Mihandoost, A. Shirazi, Z. Sepehrizadeh, J. T. Bazzaz and M. Ghazi-khansari, Melatonin may play 
a role in modulation of bax and bcl-2 expression levels to protect rat peripheral blood lymphocytes from gamma irradiation-induced apoptosis, Mutat. Res., 2012, 738-739, 19-27.

21 C. Xu, A. Wu, H. Zhu, H. Fang, L. Xu, J. Ye, et al., Melatonin is involved in the apoptosis and necrosis of pancreatic cancer cell line SW-1990 via modulating of Bcl-2/Bax balance, Biomed. Pharmacother., 2013, 67(2), 133-139.

22 V. Martin, A. M. Sanchez-Sanchez, F. Herrera, C. GomezManzano, J. Fueyo, M. A. Alvarez-Vega, et al., Melatonininduced methylation of the ABCG2/BCRP promoter as a novel mechanism to overcome multidrug resistance in brain tumour stem cells, Br. J. Cancer, 2013, 108(10), 20052012.

23 P. Dobsak, J. Siegelova, J. C. Eicher, J. Jancik, H. Svacinova, J. Vasku, et al., Melatonin protects against ischemiareperfusion injury and inhibits apoptosis in isolated working rat heart, Pathophysiology, 2003, 9, 179-187.

24 A. Laliena, B. San Miguel, I. Crespo, M. Alvarez, J. GonzálezGallego and M. J. Tuñón, Melatonin attenuates inflammation and promotes regeneration in rabbits with fulminant hepatitis of viral origin, J. Pineal Res., 2012, 53(3), 270-278.

25 Y. L. Liang, Z. H. Zhang, X. J. Liu, X. Q. Liu, L. Tao, Y. F. Zhang, et al., Melatonin protects against apoptosisinducing factor (AIF)-dependent cell death during acetaminophen-induced acute liver failure, PLoS One, 2012, $7(12)$, e51911.

26 C. Koshy, E. S. Schweikhard, R. M. Gärtner, C. Perez, O. Yildiz and C. Ziegler, Structural evidence for functional lipid interactions in the betaine transporter BetP, $E M B O J$., 2013, 32(23), 3096-3105.

27 P. M. Ueland, Choline and betaine in health and disease, $J$. Inherited Metab. Dis., 2011, 34(1), 3-15.

28 K. K. Kharbanda, S. L. Todero, B. W. Ward, J. J. Cannella 3rd and D. J. Tuma, Betaine administration corrects ethanolinduced defective VLDL secretion, Mol. Cell. Biochem., 2009, 327(1-2), 75-78.

29 K. K. Kharbanda, Alcoholic liver disease and methionine metabolism, Semin. Liver Dis., 2009, 29(2), 155-165.

30 Molinspiration Cheminformatics, http://www.molinspiration. com, Molinspiration property engine v2013.09.

31 ACD-I/Lab, https://ilab.acdlabs.com.

32 A. Smelcerovic, M. Rangelov, Z. Smelcerovic, A. Veljkovic, E. Cherneva, D. Yancheva, et al., Two 6-(propan-2-yl)-4methyl-morpholine-2,5-diones as new non-purine xanthine oxidase inhibitors and anti-inflammatory agents, Food Chem. Toxicol., 2013, 55, 493-497.

33 J. A. Buege and S. D. Aust, Microsomal lipid peroxidation, Methods Enzymol., 1978, 52, 302-310.

34 J. Bartholeyns, C. Peeters-Joris, H. Reychler and P. Baudhuin, Hepatic nucleases: 1 . Methods for the specific determination and characterization in rat liver, Eur. J. Biochem., 1975, 57, 205-211.

35 G. Kocic, R. Pavlovic, S. Najman, G. Nikolic, D. Sokolovic, T. Jevtovic-Stoimenov, et al., Circulating ribonucleic acids and metabolic stress parameters may reflect progression of autoimmune or inflammatory conditions in juvenile type 1 diabetes, Sci. World J., 2011, 11, 1496-1508.

36 O. Gortzi, S. Lala, I. Chinou and J. Tsaknis, Evaluation of the antimicrobial and antioxidant activities of Origanum dictamnus extracts before and after encapsulation in liposomes, Molecules, 2007, 12(5), 932-945.

37 N. El Moussaoui and A. Bendriss, Analysis of melatonin by high performance liquid chromatography after solid-phase extraction (SPE/HPLC-FD), Int. J. Eng. Sci. Res. Technol., 2015, 4(2), 988-993.

38 R. Storey and R. G. W. Jones, Betaine and choline levels in plants and their relationship to $\mathrm{NaCl}$ stress, Plant Sci. Lett., 1975, 4(3), 161-168.

39 C. A. Lipinski, F. Lombardo, B. W. Dominy and P. J. Feeney, Experimental and computational approaches to estimate solubility and permeability in drug discovery and development settings, Adv. Drug Delivery Rev., 2012, 64, 4-17.

40 A. P. Demchenko, The change of cellular membranes on apoptosis: fluorescence detection, Exp. Oncol., 2012, 34, 263-268.

41 T. Matsura, Oxidized phosphatidylserine: production and bioactivities, Yonago Acta Med., 2014, 57(4), 119-127.

42 T. Matsura, A. Togawa, M. Kai, T. Nishida, J. Nakada, Y. Ishibe, et al., The presence of oxidized phosphatidylserine on Fas-mediated apoptotic cell surface, Biochim. Biophys. Acta, 2005, 1736(3), 181-188.

43 A. Yamashita, H. Morikawa, N. Tajima, M. Teraoka, C. Kusumoto, K. Nakaso, et al., Mechanisms underlying production and externalization of oxidized phosphatidylserine in apoptosis: involvement of mitochondria, Yonago Acta Med., 2012, 55(1), 11-20.

44 S. Sharma, J. Sarkar, C. Haldar and S. Sinha, Melatonin reverses Fas, E2F-1 and endoplasmic reticulum stress mediated apoptosis and dysregulation of autophagy induced by the herbicide atrazine in murine splenocytes, PLoS One, 2014, 9(9), e108602.

45 M. J. Tuñón, B. San Miguel, I. Crespo, F. Jorquera, E. Santamaría, M. Alvarez, et al., Melatonin attenuates apoptotic liver damage in fulminant hepatic failure induced by the rabbit hemorrhagic disease virus, J. Pineal Res., 2011, 50(1), 38-45.

46 D. Vay, C. Rigamonti, M. Vidali, E. Mottaran, E. Alchera, G. Occhino, et al., J. Hepatol., 2006, 44, 183-189.

47 D. Graf, A. K. Kurz, R. Reinehr, R. Fischer, G. Kircheis and D. Häussinger, Prevention of bile acid-induced apoptosis by betaine in rat liver, Hepatology, 2002, 36(4), 829-839.

48 H. Dies, B. Cheung, J. Tang and M. C. Rheinstädter, The organization of melatonin in lipid membranes, Biochim. Biophys. Acta, 2015, 1848(4), 1032-1040.

49 E. Drolle, N. Kucerka, M. I. Hoopes, Y. Choi, J. Katsaras, M. Karttunen, et al., Effect of melatonin and cholesterol on the structure of DOPC and DPPC membranes, Biochim. Biophys. Acta, 2013, 1828(9), 2247-2254.

50 D. Bongiorno, L. Ceraulo, M. Ferrugia, F. Filizzola, A. Ruggirello and V. T. Liveri, Localization and interactions of melatonin in dry cholesterol/lecithin mixed reversed micelles used as cell membrane models, J. Pineal Res., 2005, 38(4), 292-298. 
51 V. R. De Lima, M. S. Caro, M. I. Tavares and T. B. CreczynskiPasa, Melatonin location in egg phosphatidylcholine liposomes: possible relation to its antioxidant mechanisms, J. Pineal Res., 2007, 43(3), 276-282.

52 G. Bekyarova, M. Tzaneva and M. Hristova, Melatonin modulates the expression of Bcl-2 family proteins in liver after thermal injury in rats, Adv. Biosci. Biotechnol., 2013, 4(11), 41-47.

53 V. Molpeceres, J. L. Mauriz, M. V. García-Mediavilla, P. González, J. P. Barrio and J. González-Gallego, Melatonin is able to reduce the apoptotic liver changes induced by aging via inhibition of the intrinsic pathway of apoptosis, J. Gerontol., Ser. A, 2007, 62(7), 687-695.

54 D. Kogel, Exploiting BH3 mimetics for cancer therapy, in Mitochondria: The anti-cancer target for the third millennium, ed. J. Neuzil, S. Pervaiz and S. Fulda, Springer, Dordrecht, Netherlands, 2014. pp. 39-58.

55 T. Akiyama, C. R. Dass and P. F. M. Choong, Bim-targeted cancer therapy: a link between drug action and underlying molecular changes, Mol. Cancer Ther., 2009, 8(12), 3173-3180.

56 K. Breitschopf, J. Haendeler, P. Malchow, A. M. Zeiher and S. Dimmeler, Posttranslational modification of Bcl-2 facilitates its proteasome-dependent degradation: molecular characterization of the involved signaling pathway, Mol. Cell. Biol., 2000, 20, 1886-1896.

57 F. Luchetti, B. Canonico, M. Betti, M. Arcangeletti, F. Pilolli, M. Piroddi, et al., Melatonin signaling and cell protection function, FASEB J., 2010, 24(10), 3603-3624.

58 R. Singh and M. J. Czaja, Regulation of hepatocyte apoptosis by oxidative stress, J. Gastroenterol. Hepatol., 2007, 22(1), S45-S48.

59 P. J. Kong, J. S. Byun, S. Y. Lim, J. J. Lee, S. J. Hong, K. J. Kwon, et al., Melatonin induces Akt phosphorylation through melatonin receptor-and PI3K-dependent pathways in primary astrocytes, Korean J. Physiol. Pharmacol., 2008, 12(2), 37-41.

60 S. I. Choi, S. S. Joo and Y. M. Yoo, Melatonin prevents nitric oxide-induced apoptosis by increasing the interaction between 14-3-3 $\beta$ and p-Bad in SK-N-MC cells, J. Pineal Res., 2008, 44(1), 95-100.

61 E. Peschke, I. Bähr and E. Mühlbauer, Melatonin and pancreatic islets: interrelationships between melatonin, insulin and glucagon, Int. J. Mol. Sci., 2013, 14(4), 6981-7015.
62 F. Nduhirabandi, E. F. Du Toit, D. Blackhurst, D. Marais and A. Lochner, Chronic melatonin consumption prevents obesity-related metabolic abnormalities and protects the heart against myocardial ischemia and reperfusion injury in a prediabetic model of diet-induced obesity, J. Pineal Res., 2011, 50(2), 171-182.

63 E. Hatano and D. A. Brenner, Akt protects mouse hepatocytes from TNF- $\alpha$ and Fas-mediated apoptosis through NK-кB activation, Am. J. Physiol.: Gastrointest. Liver Physiol., 2001, 281(6), G1357-G1368.

64 F. Radogna, S. Cristofanon, L. Paternoster, M. D'Alessio, M. De Nicola, C. Cerella, et al., Melatonin antagonizes the intrinsic pathway of apoptosis via mitochondrial targeting of Bcl-2, J. Pineal Res., 2008, 44(3), 316-325.

65 F. Radogna, M. C. Albertini, M. De Nicola, M. Diederich, I. Bejarano and L. Ghibelli, Melatonin promotes Bax sequestration to mitochondria reducing cell susceptibility to apoptosis via the lipoxygenase metabolite 5hydroxyeicosatetraenoic acid, Mitochondrion, 2015, 21, 113121.

66 C. S. Rajendrakumar, T. Suryanarayana and A. R. Reddy, DNA helix destabilization by proline and betaine: possible role in the salinity tolerance process, FEBS Lett., 1997, 410(2), 201-205.

67 G. Hatzis, P. Ziakas, N. Kavantzas, A. Triantafyllou, P. Sigalas, I. Andreadou, et al., Melatonin attenuates high fat diet-induced fatty liver disease in rats, World J. Hepatol., 2013, 5(4), 160-169.

68 R. A. Saad, M. F. EL-Bab and A. A. Shalaby, Attenuation of acute and chronic liver injury by melatonin in rats, $J$. Taibah Univ. Sci., 2013, 7(2), 88-96.

69 D. Schiller, V. Ott, R. Krämer and S. Morbach, Influence of membrane composition on osmosensing by the betaine carrier BetP from Corynebacterium glutamicum, J. Biol. Chem., 2006, 281(12), 7737-7746.

70 D. F. Veber, S. R. Johnson, H. Y. Cheng, B. R. Smith, K. W. Ward and K. D. Kopple, Molecular properties that influence the oral bioavailability of drug candidates, $J$. Med. Chem., 2002, 45(12), 2615-2623.

71 W. J. Egan, K. M. Merz and J. J. Baldwin, Prediction of drug absorption using multivariate statistics, J. Med. Chem., 2000, 43(21), 3867-3877. 